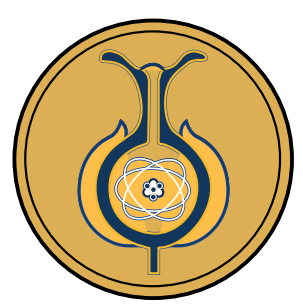

\title{
Competencias de profesores en formación en matemáticas al transformar las representaciones de una función
}

\author{
Competencies of pre-service mathematics teachers when transforming the \\ representations of a function \\ Competências de docentes em formação em matemática ao transformar as \\ representações de uma função
}

Tulio Amaya De Armas ${ }^{1}$ Arjuna Gabriel Castellanos ${ }^{2}$ Luis Roberto Pino-Fan ${ }^{3}$

Received: Jun/21/2020 • Accepted: Sep/16/2020 • Published: Jul/31/2021

\section{Resumen}

En este trabajo se tuvo como objetivo analizar las competencias de futuros profesores de pedagogía en enseñanza media en matemáticas, al hacer transformaciones de las representaciones de una función. La información se recogió en el segundo semestre de 2019 y se procesó utilizando la técnica análisis de contenido. Es un trabajo cualitativo, donde se interactuó con docentes en formación, mientras resolvían situaciones problema que involucraban funciones y preparaban una clase, que luego simulaban ante su grupo de clase y su docente. La muestra la constituyeron 36 sujetos estudiantes del profesorado en matemáticas de una universidad chilena. Los resultados muestran que lograron producir múltiples formas de representación de las relaciones funcionales analizadas, lo que facilitaba el análisis y establecimiento de conexiones con elementos del contexto sociocultural, pero una parte del grupo presentó dificultades con la fluidez perceptual, lo que impidió establecer conexiones entre ellas. Fueron modificando los significados parciales de la función, articuladamente, hasta ubicarse en uno de ellos, desde donde produjeron y articularon sus múltiples representaciones. Se concluye sobre la necesidad de implementar procesos de intervención que lleven a los futuros profesores a hacer análisis más integrales de las funciones, que le faciliten hacer un uso operativo de sus conocimientos, para minimizar las dificultades de aprendizaje en sus estudiantes.

Palabras clave: profesores en formación; funciones; registros semióticos; significados parciales de las funciones; representaciones semióticas; educación matemática.

\section{Abstract}

This paper aimed to analyze the competencies of pre-service secondary school mathematics teachers when transforming the representations of a function. The information was collected during the second half of 2019

\footnotetext{
Tulio Amaya De Armas, $\square$ tuama1@hotmail.com, (D) https://orcid.org/0000-0003-0342-4338

Arjuna Gabriel Castellanos, $\square$ acastellanosm@hotmail.com, (D) https://orcid.org/0000-0003-1702-575X

Luis Roberto Pino-Fan, $\triangle$ luis.pino@ulagos.cl, (D) https://orcid.org/0000-0003-4060-7408

1 Institución Educativa Madre Amalia de Sincelejo, Colombia

2 Institución Educativa la Milagrosa de Bello, Colombia

3 Departamento de Ciencias Exactas, Universidad de Los Lagos, Chile
} 
and was processed using the content analysis technique. For this qualitative study, researchers interacted with pre-service teachers while they solved problems involving functions and prepared a class that they later simulated before their professor and classmates. The sample included 36 students from the Mathematics Education Program of a Chilean university. Results show that pre-service teachers were able to produce multiple ways of representations of the analyzed functional relationships, which facilitated the analysis and establishment of connections with sociocultural elements. However, some participants faced difficulties with perceptual fluency, which prevented them from establishing connections. The partial meanings of the functions were articulately modified until they became one, in which time multiple representations were produced and articulated. In conclusion, interventional processes should be implemented to help future teachers make more comprehensive analyzes of functions and have an operational use of their knowledge, in order to minimize learning difficulties in students.

Keywords: pre-service teachers; function; registers of semiotic representation; partial meanings of functions; semiotic representations; Mathematics education.

\section{Resumo}

Este trabalho teve como objetivo analisar as competências de futuros professores de pedagogia no ensino médio em matemática, ao fazer transformações das representações de uma função. A informação foi coletada no segundo semestre de 2019 e processada com o uso do método de análise de conteúdo. É um trabalho qualitativo, no qual houve uma interação com docentes em formação, enquanto resolviam situações problema que envolviam funções e preparavam uma aula, que logo simulavam diante de seu grupo da sala e de seu docente. A amostra esteve composta de 36 sujeitos estudantes da faculdade de matemática de uma universidade chilena, com os quais interagiram na preparação e na simulação de uma aula sobre a noção função. Os resultados mostram que conseguiram produzir múltiplas formas de representação das relações funcionais analisadas, o que facilitava a análise e 0 estabelecimento de conexões com elementos do contexto sociocultural, porém uma parte do grupo apresentou dificuldades com a fluidez perceptual, impedindo estabelecer conexões entre elas. Foram modificando os significados parciais da função, articuladamente, até localizar-se em um deles, desde onde produziram e articularam suas múltiplas representações. Conclui-se sobre a necessidade de implementar processos de intervenção que guie os futuros professores a fazer análises mais integrais das funções, o que facilita fazer um uso operativo de seus conhecimentos e, assim, minimizar as dificuldades de aprendizagem em seus estudantes.

Palavras-chave: docentes em formação; função; registros semióticos; significados parciais das funções; representações semióticas; educação matemática

\section{INTRODUCCIÓN}

Las representaciones son el medio que facilita el acceso a los objetos matemáticos, si no se acude a ellas es imposible su estudio (Duval, 2017). Para Dreher y Kuntze (2015), la traducción de una representación a otra es un pilar fundamental para la comprensión de los objetos matemáticos. Además, el dominio del personal docente sobre múltiples formas de representación de una función y la articulación de estos distintos tipos de representación son de suma importancia en los procesos de enseñanza y de aprendizaje de 
las funciones (Even, 1990; Kurnaz y Bayri, 2018). Pues, la capacidad de coordinar dos o más representaciones es vista como un sello distintivo para el desarrollo de competencias matemáticas, y se asocia a buenos resultados académicos por la parte estudiantil (Wills, Shipley, Chang, Cromley y Booth, 2014), por lo que es de esperar que, al trabajar con funciones, se analicen múltiples representaciones de estas.

Con base en lo anterior, la orientación de un proceso de enseñanza y aprendizaje del objeto matemático función requiere, del profesorado, conocimientos y competencias en el manejo y uso de sus múltiples formas de representación (Gagatsis y Shiakalli, 2004), de tal forma que lleve a sus estudiantes a asignarles significados a los elementos de las diversas representaciones, en sus diferentes registros y a conectar los significados inter e intra registros, para que comprendan el concepto de la manera más integral posible (Amaya, 2020).

Sin embargo, estudios previos reportan una comprensión limitada de las representaciones (Dreher y Kuntze, 2015) y de las funciones (Amaya, 2020; Amaya, Pino-Fan y Medina, 2016; Biehler, 2005; Even, 1990; 1993), al resolver situaciones que las involucren, tanto en docentes en formación como en ejercicio, ya que no logran conectar las representaciones producidas, y les cuesta integrar los significados parciales de una función. Estas limitaciones en quien enseña podrían resultar nocivas para los procesos de enseñanza y de aprendizaje, ya que su capacidad para hacer transformaciones entre los elementos de las representaciones de una función condiciona el éxito o fracaso para la comprensión de esta noción por parte de sus estudiantes (Gagatsis y Shiakalli, 2004). En consecuencia, la falta de comprensión del objeto matemático función podría afectar el acceso al cálculo (Hitt, 1998) o al razonamiento algebraico en estudios más avanzados en matemáticas.

En este sentido, el objetivo de este artículo es presentar los resultados del análisis de las competencias de los futuros profesores para hacer transformaciones de las representaciones de una función. Se enfatizó en identificar el conocimiento que los profesores en formación tienen sobre las funciones; pero, sobre todo, si pueden establecer relaciones entre sus representaciones con elementos conceptuales correspondientes y con elementos socioculturales, donde se les pueda asignar significado y sentido. Darle un sentido así a las funciones, va a permitir identificarlas y comprenderlas en contexto, establecer conexiones y correspondencias entre sus elementos y diseñar tareas contextualizadas que las involucren. Además, el significado de la función, movilizado de esta manera en la solución de las situaciones, parece impactar en las características de las representaciones producidas por quienes las analizan; a su vez, el registro tomado como base para hacer el análisis de los sistemas semióticos emergentes también parece impactar las características de las articulaciones de elementos conceptuales y socioculturales correspondientes.

Para llevar a cabo el estudio, se utilizaron nociones teórico-metodológicas, principalmente de dos perspectivas teóricas propias de la didáctica de la matemática: teoría de los registros de representación semióticos (TRRS) (Duval, 2017) y el enfoque ontosemiótico (EOS) del conocimiento y la instrucción matemáticos (Godino, Font y Batanero, 2007). La articulación de elementos de ambos enfoques permite apreciar la complementariedad entre ellos, y enriquece el estudio, pues con los registros $\mathrm{y}$ representaciones se pueden hacer cierto 
análisis, que resultan más ricos al incorporar objetos matemáticos primarios como flechas, estrategias/procedimientos o conceptos/definiciones utilizados, por quien comunica una respuesta, para conectar las representaciones, lo que permite una mirada más holística del desarrollo formativo del futuro personal docente. En la siguiente sección se describen las nociones utilizadas de ambos enfoques, así como la articulación de tales nociones con las competencias y los conocimientos necesarios para que el profesorado de matemáticas gestione, adecuadamente, los aprendizajes de sus estudiantes.

\section{MARCO TEÓRICO}

\section{Competencias fundamentales en el profesorado de matemáticas}

Según Breda, Pino-Fan y Font (2017), si un profesor o profesora desea establecer ciclos de planificación, implementación, valoración y plantear propuestas de mejora en el desarrollo de su actividad didáctico-matemática, necesita dominar dos competencias fundamentales: (1) la competencia matemática, que le permite elegir y resolver tareas adecuándolas a las necesidades de aprendizaje de sus estudiantes del nivel que orienta; y (2) la competencia de análisis e intervención didáctica.

El desarrollo de la competencia (1) está relacionado con lo que Schoenfeld y Kilpatrick (2008) denominan "conocer las matemáticas con profundidad y amplitud", para lo cual se requiere del profesorado que sepa escoger tareas adecuadas al nivel estudiantil que oriente, poderlas resolver utilizando diversos procedimientos y producir diversas representaciones del objeto matemático enseñado. Se requiere, además, que vincule objetos matemáticos del nivel donde orienta los aprendizajes, con conceptos de otros niveles y que pueda particularizar o generalizar los conceptos estudiados. Lo anterior guarda relación con lo que Pino-Fan y Godino (2015) denominan 'dimensión matemática' y 'faceta epistémica' del conocimiento didáctico-matemático (CDM) del personal docente.

Por su parte, para lograr la competencia (2), de análisis e intervención didáctica, que le permite al profesorado seleccionar, adecuar, diseñar, aplicar y valorar secuencias de aprendizaje propias y ajenas, utilizando técnicas de análisis didáctico y criterios de "calidad" (Breda, Font y Pino-Fan, 2018), "debe hacer transposiciones didácticas adecuadas a quien se enseña, que los lleve a movilizar y comprender la diversidad de significados parciales para un objeto matemático en los diversos contextos en donde sea posible analizarlo" (Amaya, 2020, p. 114). Ello debe facilitarle, al profesorado, hacer diversas justificaciones y argumentaciones e identificar los conocimientos puestos en juego durante el desarrollo de una tarea matemática (Pino-Fan y Godino, 2015). Además, es propio de docentes con esta competencia, escoger los registros a utilizar, las representaciones y lo recursos que les permitan adecuar la clase a las posibilidades de comprensión de sus estudiantes. En este sentido, deben escoger el orden en que se producen las representaciones de dichos objetos; decidir qué registro utilizar como registro de partida y el orden de los registros auxiliares; articular los registros y las representaciones entre sí y con elementos conceptuales correspondientes y del contexto sociocultural; todo ello para facilitarles a sus estudiantes la asignación de significados y sentidos (Amaya, 2016).

En efecto, en diversos estudios (Godino, Giacomone, Font y Pino-Fan, 2018; 
Pino-Fan, Font y Breda, 2017) se ha presentado el modelo CDM como una 'macro herramienta' metodológica que permite caracterizar y desarrollar las dos competencias fundamentales antes comentadas. Claro, esto supone considerar la noción de competencia desde una perspectiva integral, como un conocimiento puesto en acción (Niss, 2019).

Atendiendo lo anterior, docentes matemáticamente competentes necesitan conocer en profundidad lo que enseñan, es decir, los conceptos, las formas como estos se relacionan, los recursos necesarios para lograr transposiciones didácticas apropiadas y la estructura de las actividades, para potenciar habilidades de pensamiento matemático en quien aprende. Se entiende por pensamiento matemático, el proceso mental referido a las formas en que una persona piensa en contextos matemáticos cotidianos, identificando representaciones fenomenológicas en su interacción con el medio o, como dice Schoenfeld (1992), a la forma en que las personas desarrollan una manera matemática de pensar en su acción cotidiana. Por lo que, al hablar de desarrollo de habilidades de pensamiento matemático, interesa que nos focalicemos en el pensamiento didáctico-matemático de quien orienta el proceso de aprendizaje, como una forma particular de actividad humana (Heuvel-Panhuizen, 2020) que provea las condiciones para que quien aprende logre potenciar su pensamiento matemático, de manera que le permita explotar al máximo sus habilidades matemáticas. Es decir, brindar elementos que apoyen el fortalecimiento de las formas matemáticas de pensar, al resolver una actividad cotidiana (Rosa y Orey, 2011), para que sus estudiantes aprendan matemáticas de manera intuitiva (Heuvel-Panhuizen, 2020), con iniciativas y estrategias propias, que les lleven a ser originales al comunicar los resultados de un problema matemático que se resuelva (Kaur, Wong, Govindani, 2020). Así, el estudio de la matemática debe hacerse con situaciones problema, donde pueda matematizarse la realidad y el estudiantado pueda utilizar sus conocimientos previos (Arcavi, 2020).

En estos términos, el análisis del desarrollo del pensamiento didáctico-matemático en profesores en formación, según Schoenfeld (1992), podría generar un particular interés por entender las razones, los procedimientos, las explicaciones, los argumentos, las escrituras o las formulaciones verbales que el alumnado construye para responder a una cierta tarea matemática, así como por descifrar los mecanismos mediante los cuales la cultura y el medio contribuyen en la formación de dicho pensamiento; aspecto que, reflejado en su práctica cotidiana, evidenciaría la adquisición de un buen dominio de competencia matemática (1). Teniendo en cuenta que, en este proceso, el propio pensamiento matemático se modifica constante y continuamente, el personal docente tiene la necesidad de conocer estos requerimientos para proveer las actividades que favorezcan la potenciación de habilidades de pensamiento matemático en el estudiantado.

\section{Los registros y las representaciones semióticas}

"Una representación es algo que representa una cosa ..., pero que no es la cosa misma" (Duval, 2006, p. 103). Las representaciones semióticas son el medio del que dispone una persona para exteriorizar sus representaciones mentales, es decir, con las representaciones semióticas se hacen visibles las representaciones mentales y facilitan que otros sujetos puedan acceder a ellas (Duval, 2017); además, están conformadas por un conjunto de signos asociados en 
reglas que conforman un sistema conectado, el cual permite la comunicación de una idea o la producción de nuevos conocimientos. Se puede entender un registro de representación como un contenedor que permite configurar las representaciones semióticas (Amaya, 2020). Cada registro tiene sus propias reglas, aunque estas no son exclusivas de un registro en particular, ya que algunas son compartidas por varios de ellos. Asimismo, un sistema semiótico emergente lo constituyen todos los registros y representaciones semióticas que sea posible producir de un objeto, desde donde se puede hacer un estudio integral de este, lo cual se constituye en la clave de la comprensión en matemáticas. Además, para un mismo objeto matemático, pueden existir infinitos sistemas semióticos emergentes que faciliten su estudio integral, ya que por cada situación que se contemple para el objeto es posible configurar uno.

Las representaciones de un objeto admiten dos tipos de transformaciones: (i) tipo tratamiento o por manipulación sintáctica, son las que se llevan a cabo sin abandonar el registro, se hacen por manipulación directa de los signos, siguiendo las reglas matemáticas propias del registro mismo, y (ii) tipo conversión o por elaboración semántica, aquellas que se hacen cambiando de registro, con símbolos del registro de llegada, pero basadas en las características del objeto representado (Kaput, 1987), conservando su significado. Cuando es posible poner en paralelo los elementos de dos o más representaciones, estas se dicen homogéneas, de lo contrario se dice que son heterogéneas. Cabe señalar que una representación contiene cierta, pero no toda, la información del objeto que representa (Duval, 2017), por lo que dos representaciones de un mismo objeto no necesariamente contienen la misma información, pero ellas se complementan (Duval, 2006). Por lo tanto, para que se puedan evidenciar, de forma integral, las características de un objeto matemático, hay que analizar todo el sistema semiótico emergente que se haya podido producir del objeto.

\section{Las funciones y sus registros de representación}

En el desarrollo histórico del objeto matemático función se puede apreciar cómo el estudio de relaciones entre magnitudes abre paso al surgimiento de esta noción (Rey, Boubée, Sastre y Cañibano, 2009), lo que orienta sobre cómo puede ser introducido a estudiantes de enseñanza media desde una perspectiva social. La función vista como relación de dependencia entre magnitudes variables, donde a cada valor de la variable independiente se le hace corresponder un único valor de la variable dependiente (Pino-Fan, Parra-Urrea y Castro, 2019), hace necesario que, quien oriente su enseñanza, tenga claro que el paso de las magnitudes concretas a las variables generalizadas sea el fundamento del uso de las relaciones funcionales, para contextualizar las funciones, como paso previo al acceso a las funciones de variable real (Font, 2011). Una relación funcional es una función que puede identificarse en un determinado contexto.

En este sentido, en un estudio de Pino-Fan et al. (2019), se identificaron siete contextos de uso histórico, que determinan los significados de referencia, para la noción función: i) la función como correspondencia; ii) la función como relación entre magnitudes variables; iii) la función como representación gráfica; iv) la función como expresión analítica; v) la función como correspondencia arbitraria; y vi) la función a partir de la teoría de conjuntos. 
Es preciso destacar que la noción de significado de un objeto matemático, en el EOS, atiende la relatividad del contexto en el que este es usado, y se define como el sistema de prácticas operativas y discursivas que una persona (o una institución) realiza para resolver una cierta clase de situaciones-problemas en las que dicho objeto interviene (Godino y Batanero, 1994). Además, en el EOS se consideran los objetos matemáticos como entidades emergentes de los sistemas de prácticas, considerando los siguientes objetos matemáticos primarios (entidades básicas de la actividad matemática): elementos lingüísticos (representaciones), situaciones-problema, conceptos/ definiciones, propiedades, procedimientos/ estrategias y argumentos.

En relación con las representaciones, según Sastre, Rey y Bourbée (2008), desde un punto de vista didáctico, el concepto función admite diferentes registros, de los cuales cada uno puede tener diferentes alcances y limitaciones. Para Amaya (2016), los registros más usados para representar una función o hacer modelos de relaciones funcionales son:

- Coloquial o del leguaje materno: se relaciona con la capacidad lingüística indispensable para la descripción de situaciones funcionales, así como la comunicación, interpretación y discusión de resultados, en él se pueden configurar representaciones coloquiales. Pueden darse transformaciones tipo tratamiento de un idioma a otro, estas son transformaciones estructurales, muy heterogéneas entre sí, es decir, los contenidos entre ellas son muy similares, pero no necesariamente coincidentes.
- Analítico: está íntimamente relacionado con la capacidad simbólica, principalmente con el álgebra y la modelación algebraica o analítica. En él se pueden configurar representaciones analítico-algebraicas y analítico-aritméticas.

- Cartesiano: corresponde a un conjunto de parejas ordenadas con un criterio de ordenación, donde el primer componente corresponde a los valores que toma la variable independiente y el segundo a los valores de la variable dependiente. En él se pueden configurar representaciones cartesianas.

- Gráfico: consiste en un sistema coordenado, donde se pueden hacer representaciones gráficas, correspondientes a un conjunto de puntos que finalmente las determinan.

- $\quad$ Figural: es un lienzo que sirve como contenedor a un dibujo, un esquema o una figura que modele una situación. En él se pueden hacer representaciones figurales o icónicas donde no se involucra un sistema coordenado.

- Tabular: consiste en una tabla, en la que se ubican las entradas, ya sea en las filas o en las columnas, de tal forma que el número de columnas (o filas según se ordenen), corresponda al total de cantidades que intervienen en la situación, que vienen a ser los elementos que constituyen la función. La tabla llena con la información correspondiente a la función es la representación tabular.

- Fenomenológico: son los aspectos del contexto que facilitan hacer la transposición didáctica de los conocimientos puestos en juego en una situación problema. Una representación fenomenológica la conforman los 
factores sociales ajustables a la clase de matemáticas, que permiten contextualizar elementos de la disciplina hasta bajarlos a un lugar asequible para el sujeto aprendiz.

En el estudio de las funciones, es fundamental que se establezcan conexiones entre sus múltiples formas de representación, y de estas, con las del registro fenomenológico, ya que esto permite visionar, de diferentes formas, una misma información y poderla analizar al comparar los elementos que sean evidentes tanto en unas como en otra representación, con elementos del contexto sociocultural. Una vez producidos el mayor número de representaciones semióticas posibles de la relación funcional que se esté estudiando, se identifican los elementos comunes ostensibles en cada representación, y la conexión se hace al explicar un elemento de una representación en otra, a través de los elementos conceptuales correspondientes, lo cual da razones de equivalencia entre dichos elementos, que faciliten asignarles significado al conectarlos con elementos de la representación fenomenológica.

Este análisis de los sistemas semióticos emergentes requiere de mucha fluidez perceptual de quien orienta el proceso de enseñanza y aprendizaje (Rau, Rummel y Aleven, 2017), si se quiere afectar, positivamente, la arquitectura cognitiva de quien aprende. Es decir, es necesario que el profesorado tenga la capacidad de analizar, visualmente, las propiedades de cada representación, como fundamento para relacionar sus elementos, y establecer equivalencias entre ellos. De este modo, el significado de los objetos estudiados surge de la coordinación de los elementos de varias representaciones y de las organizaciones cognitivas que el sujeto hace, al poner dichos elementos en paralelo con elementos conceptuales (Duval, 2017) y de las representaciones fenomenológicas asociadas. En este sentido, "cabe destacar el papel de las representaciones semióticas, como andamio en la adquisición de los conocimientos matemáticos" (Amaya, 2016, p. 48), pues son la base de la conexión, tanto entre registros, como de estos con elementos conceptuales y socioculturales, a los que puedan asociarse los objetos matemáticos que se pongan en juego. Pues, las competencias docentes, en la producción y articulación de registros y representaciones, con otros objetos matemáticos primarios, son herramientas que facilitan ayudar a quien aprende a hacer conexiones ricas entre los elementos del objeto estudiado, que lo pueden llevar a comprenderlo de una manera bastante integral (Arcavi, 2020; Kaur et al., 2020).

\section{ASPECTOS METODOLÓGICOS}

Se realizó un estudio cualitativo, donde se interactuó con docentes en formación, en la preparación y simulación de una clase. Fue un proceso eminentemente social, donde el conocimiento matemático se construía mientras se resolvía una situación que simula una actividad cotidiana del contexto donde se desenvuelven el estudiantado; así, los conocimientos se produjeron como resultado de este proceso interactivo (Nonaka y Toyama, 2015), sin llevar a cabo ningún taller o proceso de intervención previo, solo los contenidos de las asignaturas desarrolladas en el programa.

\section{Muestra de informantes}

La muestra de informantes la constituyeron 36 docentes en formación de un programa de Pedagogía en Enseñanza Media en 
Matemáticas de una universidad chilena. El programa tiene una duración de 9 semestres. Los futuros profesores de la muestra tenían edades entre 19 y 30 años. El grupo se eligió de forma intencional, usando como criterio de inclusión, que los participantes hubieran cursado la asignatura Didáctica de la aritmética. En esta asignatura se orientan formas de enseñar conceptos como, operaciones básicas, secuencias y progresiones, se ofrece en el programa, a inicios del segundo año de la carrera, y tiene como prerrequisitos las asignaturas Aritmética, Introducción a la didáctica e Introducción al análisis. Estos profesores en formación, al momento de recoger la información, ya habían cursado dos de las cuatro prácticas pedagógicas que se ofrecen en el programa y solo les faltaba realizar la práctica profesional.

\section{Recolección de la información}

Para recoger la información, con los profesores en formación se emprendió un proceso de búsqueda de situaciones que involucren funciones, sobre todo lineales. Se buscó en bases de datos y librerías científicas online, que albergan revistas, libros y otros materiales clasificados. Además, se le entregó una lista con situaciones elaboradas por el equipo investigador, y se le pidió ampliar la búsqueda en las bases curriculares del Ministerio de Educación (2019). Cada docente en formación llevó a la clase la lista de situaciones de su interés, de las que seleccionó una para el desarrollo de su clase y expuso las razones de su selección; tales razones referían a su preferencia por el dominio de un registro en particular, el cual escogió como registro de partida. Se pidió trabajar en grupos de tres o cuatro integrantes; estos grupos fueron armados libremente. Aquí se reportan resultados y generalidades de todas las clases, pero se dan ejemplos solo de dos de dichas situaciones, con las que trabajaron sendos grupos de estudiantes. La información se recogió en el segundo semestre de 2019. En la Tabla 1 se presentan dos de las situaciones que se trabajaron en las clases, las cuales son muy comparables al resto de las situaciones utilizadas por los demás grupos.

Tabla 1. Muestra del tipo de actividades desarrolladas

\begin{tabular}{|c|c|}
\hline Enur & \\
\hline $\begin{array}{l}\text { Juan vive en una ciudad turística donde } \\
\text { un atractivo es el transporte público en } \\
\text { bicicletas. Así que Juan trabaja de bici- } \\
\text { taxista, haciendo un recorrido fijo para } \\
\text { turistas y, cada persona, por el recorrido } \\
\text { (paseo) que se le hace, paga } \$ 700 \text {. La } \\
\text { bicicleta no es de su propiedad y le } \\
\text { tiene que entregar al dueño una tarifa } \\
\text { diaria de } \$ 12000 \text {. } \\
\text { La señora María tiene un plan de la } \\
\text { empresa de telefonía Claro consistente } \\
\text { en un cargo fijo de } \$ 8000 \text { y } \$ 70 \text { por } \\
\text { cada minuto que consuma. }\end{array}$ & $\begin{array}{l}\text { a. Encuentra los salarios diarios de Juan (el valor de la factura } \\
\text { de María), cuando hace (consume) 12, 15, 20, 42, 51, } 31 \\
\text { carreras (minutos). } \\
\text { b. Si Juan (María) quiere ganarse (pagar) en un día (mes) } \$ 17 \\
400 \text { (10 950) ¿cuántas(os) carreras (minutos) debe hacerse } \\
\text { (consumir)? } \\
\text { c. Describe cómo obtuviste la respuesta a la pregunta anterior (b.). } \\
\text { d. ¿En cuánto varía el salario diario de Juan (valor total de la } \\
\text { factura de la señora María) por cada carrera (minuto) que } \\
\text { haga (hable)? ¿Es constante o variable esa variación? } \\
\text { e. ¿Qué cantidades intervienen en la situación problema? } \\
\text { f. Euáles varían y cuáles son constantes (fijas)? }\end{array}$ \\
\hline
\end{tabular}

Nota: Elaboración propia 
En la primera consigna: "encuentra los salarios diarios de Juan (el valor de la factura de María), cuando hace (consume) 12, 15, 20, 42, 51, 31 carreras (minutos)", se pretende que el estudiantado, utilizando cualquier estrategia, logre establecer una regla recursiva que le permita encontrar un patrón de regularidad para la situación y, así, pueda predecir valores alejados de la secuencia. Este tipo de planteamiento se puede abordar desde varias perspectivas, la pregunta pudo haber sido: ¿cuántas carreras (minutos) hizo (consumió) Juan (María), si se sabe que ganó (pagó) \$-3 600 (8 840), \$-1.500 (9 050), \$2 000 (9 400), \$4 800 (10 940), \$17 400 (11 570), \$23 700 (11 570) o $\$ 9700$ (10 170), ese día (por la factura)?

La segunda opción de pregunta es más demandante, puesto que lleva a utilizar el concepto de ecuación para encontrar una incógnita, con lo que, tradicionalmente, el estudiantado presenta dificultades: pues tiende a utilizar estrategias de tanteo o desarrollos paso a paso, hasta encontrar un término alejado de una secuencia; pero estas son cuestiones por las que se indaga en el cuestionario. Justamente, un buen ejercicio para el personal docente consiste en combinar los valores involucrados en las preguntas que lleven a las mismas opciones de respuestas y obtener nuevas preguntas.

En la consigna (c) "describe cómo obtuviste la respuesta a la pregunta anterior (b)", se indaga sobre la capacidad estudiantil para describir, en el lenguaje materno o estructural, los procedimientos realizados para tratar de dar con una respuesta determinada; es decir, al estudiantado se le pide armonizar los elementos de la representación coloquial o estructurales, con la representación del registro que haya tomado como auxiliar para hacer las conversiones o tratamientos que le permitan dar sus respuestas. Este tipo de respuestas le permiten, al profesorado de matemáticas, analizar los procesos argumentativos dados por sus estudiantes, de la forma como comunican sus respuestas, es decir, la forma de comunicar tanto las explicaciones de los procesos realizados, como las justificaciones o razones del porqué realizan un determinado procedimiento y no otro.

En la siguiente consigna: ¿en cuánto varía el salario diario de Juan (valor total de la factura de la señora María) por cada carrera (minuto) que haga (hable)? ¿Es constante o variable esa variación?, se pretende que el estudiantado haga un análisis inductivo hasta obtener la razón de cambio de la relación funcional, que le permita inferir que esa variación es constante. Que el estudiantado llegue a hacer este tipo de inferencias le puede permitir, a su docente de matemáticas, abordar, con mayor facilidad, el concepto de pendiente de una recta.

Además, se pide encontrar: "¿qué cantidades intervienen en la situación? ¿Cuáles varían y cuáles son constantes (fijas)?”. Con estas preguntas se pretende que identifiquen y clasifiquen los elementos de la relación funcional, es decir, se indaga por la capacidad para identificar y clasificar los elementos de una función y la relación de dependencia que existe entre las variables involucradas en la situación. Este tipo de consignas son fundamentales en el análisis de una relación funcional, puesto que al responder estas preguntas se obtienen los elementos de la función en el registro fenomenológico, que son los que, al armonizarlos en las diferentes representaciones de la función involucrada, van a permitir asignar significado y sentido al objeto matemático función.

Cuando se pide: "escribe una expresión matemática que modele la situación", se pretende que el estudiantado produzca representaciones en los registros aritmético 
algebraico, aritmético numérico, tabular, gráfico, o en cualquier otro. Con el análisis de los elementos de las representaciones producidas y su armonización con los elementos correspondientes del registro fenomenológico, se pone al estudiantado a asignar significado y sentido a los objetos matemáticos estudiados.

En la Tabla 2 se presentan las categorías, los indicadores y los análisis proyectados para el análisis de las clases simuladas por los futuros profesores en el desarrollo de esta actividad.

\section{Procesamiento de la información}

Tanto la información oral como la escrita se procesó con la técnica análisis de contenido (Bernárdez, 1995). La información se iba resumiendo en una matriz de valoración, de la cual se hicieron agrupaciones de las ideas básicas, siguiendo criterios temáticos (Mora, Riquelme, Troncoso y Escobar, 2018), de donde también se tomaron elementos, tanto para retroalimentar el proceso sobre la marcha, como para hacer la descripción de los resultados y analizarlos frente a los referentes teóricos que fundamentan la investigación. Además, las etiquetas $\mathrm{Gi}$, con $\mathrm{i}=1,2, \ldots, 10$, se utilizan para denotar los grupos de trabajo en los que se subdividieron los 36 docentes participantes en nuestro estudio.

\section{RESULTADOS Y ANÁLISIS DE LA INFORMACIÓN}

Respecto de la primera categoría de análisis, se pudo apreciar que hubo muchos aspectos comunes en el desarrollo de todas las clases. Todos los profesores en formación escogieron situaciones, con relaciones funcionales, adecuadas al grado y contexto

Tabla 2. Categorias y criterios para el análisis

\begin{tabular}{|c|c|c|c|}
\hline Categorías de análisis & & Indicadores para el análisis & Análisis proyectado \\
\hline $\begin{array}{l}\text { 1. Características de la } \\
\text { tarea y las soluciones } \\
\text { esperadas }\end{array}$ & b) & $\begin{array}{l}\text { Tipo de situaciones propuestas y los } \\
\text { contextos en que estas se dan. } \\
\text { Significados que se espera movilizar } \\
\text { en la escogencia de las situaciones en } \\
\text { función de los contextos. }\end{array}$ & $\begin{array}{l}\text { Identificación y análisis de } \\
\text { los elementos de la función } \\
\text { mostrados en el desarrollo de } \\
\text { la clase. }\end{array}$ \\
\hline $\begin{array}{l}\text { Características de los } \\
\text { registros y representa- } \\
\text { ciones producidas }\end{array}$ & a) & $\begin{array}{l}\text { Identificación y uso del objeto matemáti- } \\
\text { co primario al que se hace referencia: } \\
\text { conceptos/definiciones, propiedades/ } \\
\text { proposiciones, estrategias/procedimien- } \\
\text { tos o argumentos. } \\
\text { Significado de la función que se moviliza } \\
\text { en la solución de la tarea. }\end{array}$ & $\begin{array}{l}\text { La relación que se establece } \\
\text { entre los significados parciales } \\
\text { de función movilizados en } \\
\text { la solución, con los objetos } \\
\text { matemáticos primarios usados } \\
\text { para desarrollar la clase. }\end{array}$ \\
\hline $\begin{array}{l}\text { Características de los } \\
\text { sistemas semióticos } \\
\text { emergentes producidos }\end{array}$ & b) & $\begin{array}{l}\text { Producción de diferentes registros y } \\
\text { representaciones e identificación de sus } \\
\text { elementos. } \\
\text { Conexiones entre representaciones, y } \\
\text { de éstas con elementos conceptuales y } \\
\text { socioculturales correspondientes. } \\
\text { Conexión del tema con otros temas del } \\
\text { currículo. }\end{array}$ & $\begin{array}{l}\text { Las conexiones establecidas } \\
\text { entre elementos de los reg- } \\
\text { istros y las representaciones } \\
\text { producidas con elementos } \\
\text { conceptuales y socioculturales. }\end{array}$ \\
\hline
\end{tabular}

Nota: Elaboración propia. 
sociocultural donde debía desarrollarse el proceso de enseñanza y aprendizaje, y presentó adecuadamente los objetivos de la clase. Asimismo, las herramientas matemáticas computacionales las utilizaron solo para presentar diapositivas o en el diseño y ejecución de actividades evaluativas, más no como recurso didáctico para desarrollar representaciones dinámicas, donde se pudieran visualizar, simultáneamente, múltiples representaciones, que facilitarían el análisis de un mismo parámetro en todas ellas.

Al igual que en Even (1990), gran cantidad de los profesores en formación $(60,7 \%)$ ve las funciones de manera

\begin{tabular}{|c|c|c|c|c|c|}
\hline Diq & $\begin{array}{l}\text { Tarrifa por } \\
\text { carreva(p) }\end{array}$ & $\left|\begin{array}{|}\text { Gantidad de } \\
\text { carrenos (c) }\end{array}\right|$ & $\left|\begin{array}{l}\text { Dinero que } \\
\text { recibeos out }\end{array}\right|$ | & $\begin{array}{l}\text { tarifa } 49 \text { puga } \\
\text { por la brcicletait) }\end{array}$ & $\begin{array}{l}\text { Solario } \\
(S=D \cdot T)\end{array}$ \\
\hline 1 & 700 & 12 & $7 w 0-128+00$ & 12 ons & $\$ .3600$ \\
\hline 2 & $+\infty 0$ & is & $7 \infty 0 \cdot 15=10.500$ & 12000 & $\$-1.500$ \\
\hline 3 & 700 & 51 & 700.51 .35700 & 12000 & $\$ 23700$ \\
\hline 4 & too & $2 y$ & $700-2 y=168 x 0$ & 12.000 & $\$ 4800$ \\
\hline 5 & too & 31 & $7+\infty 5 i=21700$ & 12.000 & $\$ 9700$ \\
\hline 6 & 700 & 42 & $700 \cdot 4<=29400$ & 12.000 & $\$ 17400$ \\
\hline * & טt & 0 & $700.0=0$ & 12000 & $\$ 12000$ \\
\hline
\end{tabular}

$$
\begin{aligned}
3-17+40 & =D-T \\
D & =77400+12000 \\
D & =21.400 \\
P C & =29400 \quad D=P \cdot C \\
C & =\frac{29400}{700} \\
C & =42
\end{aligned}
$$$$
\text { 4-sabemas que }
$$$$
23 .+\infty>17.400
$$$$
\text { necesita } 42 \text { carreras para ganar } 17 \text { too }
$$

$$
\text { comprefbo }
$$

$$
42.700
$$$$
29+00
$$$$
\begin{aligned}
& 29400 \\
& 12000 \\
& \hline 17.400
\end{aligned}
$$

$$
\begin{array}{r}
23.700 \\
-17.400 \\
\hline 6.300
\end{array}
$$

$$
\begin{aligned}
& \text { reresitumos más } \\
& 6300=c \cdot 700 \\
& c=\frac{6300}{700}
\end{aligned}
$$$$
\text { arrems }
$$$$
c=9 \text {. }
$$

$9+42.51=$ carrerus que necesita reallear * Intersección con el ele $y$ = Tarifa diaria

Figura 1. Solución dada por G2 a la situación desarrollada en su clase. puntual, es decir, no las conciben en su integralidad; un ejemplo de ello es el análisis hecho de los intervalos de variación, los que solo se analizaron parcialmente, sin llegar a concebirlos de forma global. Como consecuencia de este análisis parcial, al comunicar el dominio y el rango, como en Amaya (2020), terminaban analizando el dominio y el rango de la función de variable generalizada asociada, es decir, $D_{A}=[-\infty,+\infty]$, sin considerar que el dominio no contiene valores negativos, pero que tampoco es infinito. Para el rango hicieron algo similar, al omitir que las relaciones funcionales con las que trabajaban tienen rangos discretos; mientras que las gráficas realizadas por el grupo eran continuas $\mathrm{y}$ tanto su dominio como su rango estaban definidos en el conjunto de los números reales. Sin embargo, un $60 \%$ contempló el cero entre los valores del dominio y valores negativos para el rango, valores que interpretó adecuadamente, como se puede apreciar en la solución comunicada por $\mathrm{G}_{2}$ (Figura 1), aspecto muy positivo, si se tiene en cuenta que la humanidad tardó siglos en concebir el cero y los números negativos (Maz, 2005).

Particularmente, $G_{2}$ y $\mathrm{G}_{10}$, en sus soluciones produjeron múltiples formas de representación, como tabulares, analíticas y gráficos; pero, similar a lo reportado por Amaya et al. (2016), parece que para $G_{2}$ y $G_{10}$, cuando se trabaja con funciones, las respuestas deben 
derivarse de sus representaciones analíticas. Un ejemplo es la solución de $\mathrm{G}_{2}$ (Figura 1), quienes debieron hacer procedimientos analíticos para encontrar el número de carreras realizadas para que el salario de Juan fuera de $\$ 17400$ y $\$ 23700$ respectivamente, cuando pudieron dar respuesta utilizando los resultados obtenidos previamente en la representación tabular. Estos mismos subgrupos $\left(\mathrm{G}_{2}\right.$ y $\left.\mathrm{G}_{10}\right)$, para ilustrar el intercepto al origen en el eje $Y$, hicieron uso de la representación aritmético-algebraica, con lo cual muestran que el término independiente corresponde a tal intercepto, sin llegar a mencionar la representación gráfica, que ya habían producido, y se prestaba para un apoyo visual muy contundente (Elia, Gagatsis y Demetriou, 2007). Asimismo, todos los subgrupos, al encontrar una expresión matemática que modelara la situación, buscaron la expresión algebraica correspondiente, como si la única forma de modelar fuera la algebraica, con lo que confunden modelación con algebrización.

Sobre la segunda categoría de análisis es posible observar en la solución de las situaciones propuestas los futuros profesores, que fueron articulando sucesivamente varios significados parciales de la noción función. Se identificaron tres formas de actuar bien diferenciadas: (1) Los subgrupos $\mathrm{G}_{1}, \mathrm{G}_{7}$ y $\mathrm{G}_{9}$, lo primero que intentaron hacer fue utilizar la definición de función desde el punto de vista conjuntista, el problema surgió al intentar dar significado a los elementos de la relación funcional, para de esta manera modelarla funcionalmente a partir de elementos del contexto. En este proceso de expresar la definición conjuntista como correspondencia arbitraria, construyeron tablas y gráficas, y el estudio de valores cambiantes de diferentes magnitudes los condujo a una primera aproximación de ciertas relaciones funcionales, al pasar de una simple tabulación de datos empíricos a la búsqueda de regularidades, y en estas, la existencia de un cierto instinto de funcionalidad (Pino-Fan et al., 2019). Seguidamente, encontraron una regla recursiva y produjeron las representaciones algebraicas correspondientes, hicieron tablas más consistentes, que contenían todas (o casi todas) las cantidades que intervienen en la situación, pero, con las representaciones producidas, no establecieron conexiones entre los elementos conceptuales y socioculturales identificables en ellas.

(2) Los grupos $G_{4}, G_{2}$ y $G_{10}$, por su parte, usaron desde el inicio el significado de la función como relación entre variables, el primer registro auxiliar que utilizaron fue el algebraico y, desde este, produjeron representaciones tabulares, gráficas, analítico-aritméticas y cartesianas. Además, establecieron correspondencias entre las características de un conjunto dado de objetos, que cada grupo identificó en su situación, con los de una secuencia numérica, producida al identificar y ejecutar un patrón y, así, comenzaron a aparecer claras manifestaciones características de la noción función (Sastre, Rey y Boubée, 2008). Por último, articularon adecuadamente algunos elementos de sus representaciones, como puede apreciase en la solución de $\mathrm{G}_{2}$ mostrada en la Figura 1. Para estos subgrupos $\left(\mathrm{G}_{4}, \mathrm{G}_{2} \mathrm{y}\right.$ $\mathrm{G}_{10}$ ), los registros y representaciones producidos (TRRS) les resultaron insuficientes para establecer conexiones de los elementos de la relación funcional que cada subgrupo analizó, con elementos conceptuales y socioculturales, por lo cual debieron recurrir a otros elementos matemáticos primarios (del EOS), como flechas, definiciones y proposiciones, con los que finalmente lograron articular tales elementos. 
(3) Otra secuencia de significados la hicieron los subgrupos $\mathrm{G}_{6}$ y $\mathrm{G}_{5}, \mathrm{G}_{3}, \mathrm{G}_{7}$ y $\mathrm{G}_{8}$, quienes en su solución iniciaron con la función como representación gráfica; $\mathrm{G}_{5}$ y $G_{7}$ usaron la representación gráfica para explicar la definición de función, utilizando el criterio de la recta vertical, y, con elementos de esta representación, encontraron con facilidad representaciones analítico-algebraicas. Una vez producida la representación aritmético-algebraica, se les facilitó la producción de otras representaciones como tabulares y cartesianas. Con la representación gráfica de base y las otras representaciones producidas, se les facilitó hacer las conexiones entre elementos conceptuales y socioculturales. Por su parte, $G_{3}, G_{6}$ y $\mathrm{G}_{8}$ explicaron la definición de función al generar una secuencia de punto, es decir, una representación cartesiana. Parece que el uso del significado de la función como representación gráfica, por sus características visuales, les facilitó a estos grupos de docentes en formación hacerse una imagen más integral de la noción función, pues fue desde esta representación que pudieron conectar elementos conceptuales y socioculturales correspondientes. Esto, según Siegler et al. (2010), tiene su explicación en que las formas gráficas, como la recta numérica, el plano cartesiano o una secuencia de puntos, dan la posibilidad de acceso a características visuales del objeto, las cuales resultan adecuadas para ilustrar mediciones y conceptos que no son fáciles de percibir en otras representaciones. Estos subgrupos también debieron apoyarse en otros elementos matemáticos primarios, y además del uso de registros y representaciones, para establecer las conexiones entre los elementos de la relación funcional analizada, utilizaron flechas y proposiciones, para hacer las conexiones entre las representaciones.
Sobre la tercera categoría de análisis, podemos señalar que cada docente en formación pudo producir más de dos representaciones de la función que analizaba, y el 70 \% logró establecer conexiones entre sus elementos. De acuerdo con Rau et al. (2017), esto permite inferir que comprenden las representaciones, al ser capaces de reproducirlas, y que $30 \%$ tiene dificultades con la fluidez perceptual (Rau et al., 2017), que impide establecer conexiones entre ellas. Lo anterior lleva a pensar que, como en Amaya (2020), este grupo $(30 \%)$ en formación tiene una comprensión limitada de las funciones, ya que, según Even (1990), poder conectar las representaciones de una función es fundamental para comprenderlas.

En términos generales, se notó en todos los futuros profesores del estudio, contrario a lo reportado por Adu-Gyamfi y Bossé (2014), que no poseen nociones variadas y desconectadas para los significados de la noción función $\mathrm{y}$, aunque cada vez que utilizaba un significado diferente asociaba la noción función con las representaciones que de esta lograba producir, el $70 \%$ articuló dichos significados con elementos conceptuales y socioculturales pertinentes. Ambos aspectos son fundamentales para comprender las funciones, puesto que entender un concepto en una representación no necesariamente significa que se comprenda en otras, por lo que es indispensable que el análisis de este concepto se haga utilizando el mayor número de representaciones que de él se logren producir (Even, 1990). Esto, teniendo en cuenta que quienes van a ensenar las funciones deberían poder producir y conectar el mayor número de representaciones de estas para optimizar su comprensión (Amaya, 2020), ya que se espera que cada docente entienda el concepto matemático para que pueda orientar su aprendizaje 
adecuadamente. Cada representación provee información diferente a la de las demás y ninguna de ellas contiene toda la información del objeto que representa (Duval, 2017), por lo que es necesario analizar el mayor número de dichas representaciones y conectarlas, para poderlas comprender de la manera más integral posible (Adu-Gyamfi y Bossé, 2014). Asimismo, las experiencias de quien aprende, con múltiples formas de representaciones, pueden permitirle construir una imagen sinérgica completa del objeto matemático que se estudia, que le facilite comprender la noción función, a partir de la integración de sus representaciones (Amaya, 2020). Estos son aspectos que denotan fortalezas y limitaciones en el conocimiento matemático especializado para la enseñanza en este grupo de docentes en formación (Ball, Thames y Phelps, 2008), pues el $70 \%$ integra con facilidad y al 30 $\%$ todavía le cuesta integrar el conocimiento didáctico con el conocimiento matemático que posee, desde donde pueda llevar a cabo situaciones de enseñanza.

Cabe señalar que los profesores en formación que pudieron producir y usar múltiples representaciones, y establecer conexiones entre ellas lograron integrar, de manera natural, los diferentes elementos ostensibles en ellas (Rau et al., 2017), como se puede apreciar en el manuscrito de $\mathrm{G}_{4}$ mostrado en la Figura 2. $G_{4}$ produjo varias representaciones, clasificaron y pudieron conectar los elementos de la función que analizaban, integrando las representaciones entre sí y con elementos del contexto sociocultural, donde les asignaron significado; un ejemplo de ello es cuando dicen: "cargo fijo $\rightarrow$ 8000 constante" ... "intercepto al origen: cargo fijo", y señala el intercepto en la ordenada al origen; "70: pendiente $\rightarrow$ es el valor del minuto" ... " $m \rightarrow$ cantidad de minutos". Este proceso de integración de las

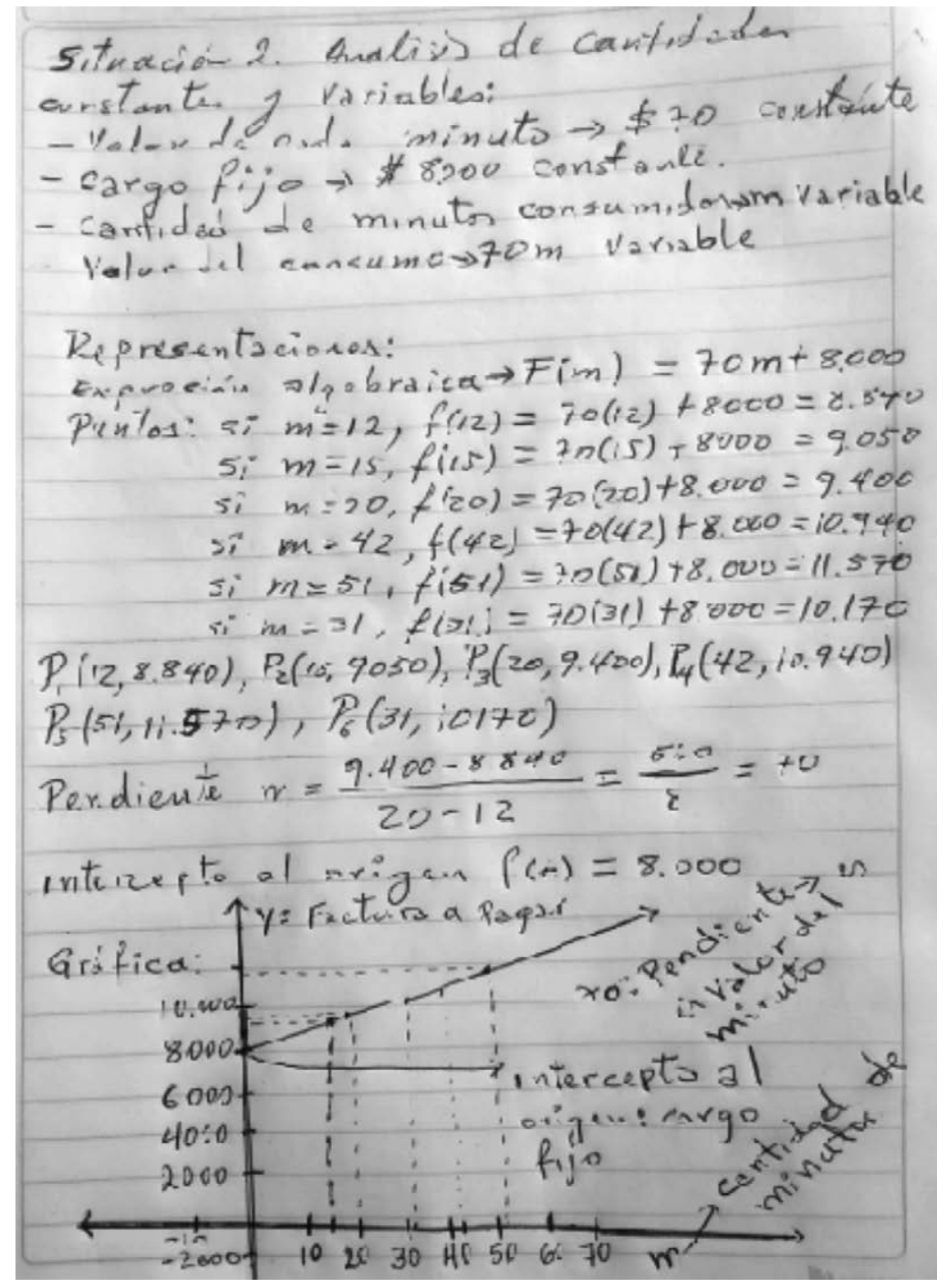

Figura 2. Solución dada por G4 a la situación desarrollada en clase. 
representaciones de una función facilita que el profesorado pueda ayudar a sus estudiantes a establecer conexiones cognitivas ricas, que apoyarán su aprendizaje matemático posterior (Gagatsis y Shiakalli, 2004), ya que la comprensión de la estructura de múltiples representaciones facilitará que el estudiantado logre una mejor aproximación al significado de algo abstracto (Duval, 2017).

Respecto del indicador (a) de la categoría de análisis 2 , podemos señalar que los elementos lingüísticos utilizados por los futuros profesores fueron mayoritariamente verbales, combinados con números, signos de operación y de agrupación, gráficos, flechas, expresiones algebraicas, tablas, parejas ordenadas y polinomios aritméticos. Entre los conceptos/definiciones usados están la de función, intercepto al origen en el eje Y, y pendiente de una recta (ver Figura 2). Los procedimientos/estrategias utilizados fueron mayoritariamente algebraicos y visuales; y entre las propiedades/proposiciones se evidencia el reconocimiento del intercepto al origen en el eje Y, en las gráficas realizadas $\mathrm{y}$ en las representaciones algebraicas y, en esta última representación, la identificación de la pendiente de la recta. Además, los argumentos utilizados contienen muy buenas explicaciones y, en la mayoría de los casos, buenas justificaciones del porqué de su actuar. Parte de los argumentos utilizados por el grupo $\mathrm{G}_{4}$ en el desarrollo de su clase son los siguientes: "La función que escogimos para nuestra clase es una función afin, pues su gráfica no pasa por el origen y su fórmula es la suma de una constante con una función lineal" ..." Al analizarla detalladamente, de manera gráfica o simbólica, se puede determinar que el cambio de un intervalo a otro es constante, y que el término independiente es el cargo fijo: 8, porque es el punto donde la gráfica corta al eje Y'. En los argumentos dados por $\mathrm{G}_{4}$, presentan los elementos de la función afín que seleccionaron, justificando las características de sus parámetros, al articular elementos de los registros gráfico, algebraico y coloquial, con elementos conceptuales y socioculturales. Este análisis requiere mucha fluidez perceptiva por parte de quien lo hace, pues para articular los elementos de varias representaciones de una función se necesita una gran habilidad de análisis visual, que facilite un entendimiento global de este concepto (Rau et al., 2017), para explorar las propiedades de cada representación e identificar elementos equivalentes en cada una de ellas. Del desarrollo de este análisis de los sistemas semióticos emergentes surgen los significados de los objetos estudiados, pues, resultan de la puesta en común de los elementos de las diferentes representaciones y la coordinación de las organizaciones cognitivas (Duval, 2017) de quien aprende, por lo que es de suma importancia que quien oriente los procesos de enseñanza y aprendizaje sea consciente de hacerlos.

Atendiendo lo anterior, hubo un grupo amplio (70\%), en quienes se evidenciaron competencias matemáticas bastante bien desarrolladas para enseñar funciones, es decir, han logrado desarrollar habilidades de pensamiento didáctico-matemático, con las que le pueden ayudar a sus estudiantes, en el fortalecimiento de elementos que apoyen las formas matemáticas de pensar al resolver una actividad cotidiana (Rosa y Orey, 2011; Schoenfeld, 1992), que involucre funciones. Como en Biehler (2005), los futuros profesores lograron concebir y utilizar aplicaciones de las funciones fuera del ámbito disciplinar de las matemáticas, utilizando relaciones funcionales, para lograr integrar varios significados parciales de la noción función, en el desarrollo de su clase. En contraste, otro grupo más reducido (30\%) mostró una visión 
limitada de las funciones, pues su formación no evidencia tener desarrollado un sistema integrado de significados parciales para esta noción, ya que estos son exhibidos como elementos individuales y no como una red de significados altamente interrelacionados, que les sirva como base para enseñar funciones (Biehler, 2005; Even, 1990, 1993).

Similar a lo reportado por Amaya (2020), el grupo de docentes en formación, en general, parece tener mejor desarrollada la competencia matemática (1), que la competencia de análisis e intervención didáctica (2). Respecto a la competencia matemática (1), como en Deulofeu (2001), la totalidad de participantes estableció conexiones entre la noción función con otros temas del currículo, por ejemplo, $G_{1}, G_{2}, G_{3}, G_{7}$ y $G_{9}$ la mostraron como indispensable para el desarrollo de conceptos posteriores, como límite de una función, derivadas e integrales. Mientras que $\mathrm{G}_{4}, \mathrm{G}_{5}, \mathrm{G}_{6}, \mathrm{G}_{8}$ y $\mathrm{G}_{10}$ evidenciaron en las representaciones analíticas producidas los elementos aditivos y multiplicativos que las componen; $\mathrm{G}_{2}, \mathrm{G}_{3}$ y $\mathrm{G}_{4}$ también las asociaron con proporcionalidad directa e inversa y establecieron diferencias entre función lineal y función afín. Además, todo el grupo enfatizó la necesidad de identificar y clasificar los elementos de la función analizada, determinando las cantidades que varían y las que permanecen fijas y estableciendo relaciones de dependencia entre las variables. Respecto a la competencia de análisis e intervención didáctica (2), un 70 $\%$ mostró avances significativos en la articulación de las representaciones producidas y en la integración de los significados parciales de la noción función, lo cual, según Ball et al (2008), lo habilita para perfilar potenciales actividades de enseñanza, pero un $30 \%$ mostró ciertas limitaciones con el dominio de estos elementos.

\section{CONCLUSIONES}

Luego del análisis de la información, se puede concluir que un grupo significativo de los profesores en formación mostró tener amplio dominio de los conocimientos matemáticos básicos que deben compartir con sus estudiantes de enseñanza media (conocimiento común del $\mathrm{CDM}$ ), así como de los conocimientos con los que estos se relacionan en niveles anteriores y posteriores (conocimiento ampliado del CDM). Utilizó variadas estrategias de solución al resolver la actividad propuesta, y se le facilitó proyectar los contenidos en relación con su utilidad, como requisito para la comprensión de otros temas posteriores, y en algunos casos, con otros temas del currículo.

Este grupo de docentes en formación mostró gran versatilidad en el manejo de herramientas computacionales, pero fue pobre su gestión y manejo de dichos recursos como herramientas didácticas que facilitaran adecuar los conocimientos al nivel estudiantil de enseñanza media. En este sentido, utilizó programas donde armó y ejecutó actividades evaluativas, más no como herramientas matemáticas computacionales como podría esperarse.

Entre los aspectos que permiten destacar la pertinencia del marco teórico utilizado, por su utilidad en el desarrollo de las clases están:

- Que el énfasis en el uso de múltiples formas de representación hizo emerger diferentes formas de simbolismo, pero los registros y representaciones resultaron insuficientes para esquematizar los elementos de las funciones, por lo que requirieron otros elementos matemáticos primarios para significar conexiones entre los elementos de las representaciones, lo que les 
facilitó hacer análisis más completos y articulados.

- $\quad$ Con respecto a la variación en la motivación de los futuros profesores por el uso de representaciones para resolver problemas matemáticos, se muestra un alto interés en las representaciones, pues advierten que aprender usándolas es agradable y entretenido, y les da cierto estatus, ya que, al lograr coordinar varias de ellas, los muestra con altas competencias matemáticas ante sus pares (Wills et al., 2014).

- $\quad$ El análisis de funciones a través de sus significados parciales y sus múltiples formas de representación hizo que los sujetos participantes interrelacionaran contenidos matemáticos con sus competencias para modelar funciones y para articular sus representaciones. En este proceso, dejaron ver tanto sus fortalezas como sus debilidades formativas. Ningún subgrupo utilizó un solo significado de función, y todos usaron variadas representaciones para desarrollar su clase. Sus dificultades fueron con la competencia análisis e intervención didáctica (2), pues les costó establecer vínculos entre las representaciones que habían producido, por lo que es necesario realizar un trabajo integral que les ayude a hacer análisis más globales de integración de estos componentes.

- $\quad$ El uso del significado de la función como relación entre magnitudes variables, con la representación algebraica como principal registro auxiliar, facilitó la producción de múltiples formas de representación de las relaciones funcionales estudiadas.

- El uso del significado de función como representación gráfica les facilitó descifrar información tanto local como global, pues todos los subgrupos que pudieron conectar los elementos de la relación funcional correspondiente lograron vincular la representación fenomenológica con la gráfica $\mathrm{y}$, a partir de esto, articular elementos de las representaciones producidas y asignarles significado y sentido, estableciendo equivalencias pertinentes entre elementos conceptuales y socioculturales.

- $\quad$ El uso de situaciones problema contextualizadas se convirtió en un detonador (Scherer, 2020) |para las producciones de los futuros profesores, pues el desafío de experimentar las matemáticas como una actividad humana (Heuvel-Panhuizen, 2020) les facilitó desarrollar su sentido común y utilizar diversas estrategias de solución a una misma tarea, a través de las cuales pudieron explicitar sus ideas y comunicarlas con sus propias palabras. Esto, según Even (1993), facilita que los maestros y maestras propicien entornos de aprendizaje que fomenten, en su alumnado, la construcción de conceptos matemáticos poderosos, conectados con sus conocimientos previos y con los contextos que comparten en comunidad.

Los resultados evidencian algunas limitaciones en el análisis e integración de los elementos de los sistemas semióticos emergentes de una función, que faciliten hacer un uso operativo de su conocimiento sobre funciones, que les favorezcan al minimizar dificultades de aprendizaje en sus estudiantes, a través del diseño y uso de situaciones problema contextualizadas. 


\section{RECONOCIMIENTOS}

Este trabajo ha sido parcialmente desarrollado en el marco del Proyecto de Investigación Fondecyt 1200005, financiado por la Agencia Nacional de Investigación y Desarrollo (ANID) de Chile.

\section{DECLARACIÓN DE LA CONTRIBUCIÓN DE LOS AUTORES}

El porcentaje total de contribución para la conceptualización, preparación y corrección de este artículo fue el siguiente: T.A.D. $40 \%$, A.G.C. $30 \%$ y L.R.P. $30 \%$.

\section{DECLARACIÓN DE DISPO- NIBILIDAD DE LOS DATOS}

Los datos que respaldan los resultados de este estudio serán puestos a disposición por el autor correspondiente [T.A.D].

\section{BIBLIOGRAFIA}

Adu-Gyamfi, K., \& Bossé, M. (2014). Processes and reasoning in representations of linear functions. International Journal of Science and Mathematics Education, 12, 167-192. https:// doi.org/10.1007/s10763-013-9416-x

Amaya, T. (2016). Evaluación de los conocimientos didáctico-matemáticos de futuros profesores de matemáticas al hacer transformaciones de las representaciones de una función [Tesis doctoral]. Universidad Nacional de Educación a Distancia, Madrid, España.

Amaya, T. (2020). Evaluación de la faceta epistémica del conocimiento didáctico- matemático de futuros profesores de matemáticas en el desarrollo de una clase utilizando funciones. Revista Bolema, 34(66), 110-131. https://doi. org/10.1590/1980-4415v34n66a06

Amaya, T., Pino-Fan, L., \& Medina, A. (2016). Evaluación del conocimiento de futuros profesores de matemáticas sobre las transformaciones de las representaciones de una función. Revista Educación Matemática, 28(3), 111-144.

Arcavi, A. (2020). Learning to Look at the World Through Mathematical Spectacles A Personal Tribute to Realistic Mathematics Education. In M. Heuvel-Panhuizen (Ed.), International Reflections on the Netherlands Didactics of Mathematics Visions on and Experiences with Realistic Mathematics Education (pp. 83-95). Springer Open.

Ball, D., Thames, M., \& Phelps, G. (2008). Content Knowledge for Teaching. What Makes It Special? Journal of Teacher Education, 59(5), 389-407. https://doi. org/10.1177/0022487108324554

Bernárdez, E. (1995). El papel del léxico en la organización textual. Universidad Complutense de Madrid.

Biehler, R. (2005). Reconstruction of Meaning as a Didactical Task: The Concept of Function as an Example. In J. Kilpatrick, C. Hoyles, O. Skovsmose \& P. Valero (Eds.), Meaning in mathematics education (pp. 61-81). Springer. https://doi.org/10.1007/0-387-24040-3_5

Breda, A., Font, V., \& Pino-Fan, L. (2018). Criterios valorativos y normativos en la didáctica de las matemáticas: El caso del constructo idoneidad didáctica. Bolema, 32(60), 255-278. http://dx. doi.org/10.1590/1980-4415v32n60a13.

Breda, A., Pino-Fan, L., \& Font, V. (2017). Meta didactic-mathematical knowledge of teachers: criteria for the reflection and assessment on teaching practice. Eurasia Journal of $\mathrm{Ma}$ thematics, Science \& Technology Education, 13(6), 1893-1918. https://doi.org/10.12973/ eurasia.2017.01207a

Deulofeu, J. (2001). Las funciones en la educación secundaria: ¿Para qué?, ¿cómo? aportaciones de la investigación. X Jornadas para la Enseñanza y el Aprendizaje de las Matemáticas, X JAEM, Zaragoza. Ponencia P41, 367-377. http://www.quadernsdigitals.net/datos/hemeroteca/r_40/nr_458/a_6226/6226.pdf.

Dreher, A., \& Kuntze, S. (2015). Teachers' professional knowledge and noticing: The case of multiple representations in the mathematics classroom. Educational Studies in Mathematics, 88(1), 89-114. https://doi.org/10.1007/ s10649-014-9577-8 
Duval, R. (2006). A cognitive analysis of problems of comprehension in a learning of mathematics. Educational Studies in Mathematics, 61, 103-131. https://doi.org/10.1007/ s10649-006-0400-z

Duval, R. (2017). Semiosis y pensamiento humano. Registros semióticos y aprendizajes intelectuales, ( $2^{\mathrm{da}}$ ed.). Universidad del Valle.

Elia, I., Gagatsis, A., \& Demetriou, A. (2007). The effects of different modes of representation on the solution of one-step additive problems. Learning and Instruction, 17, 658-672. https:// doi.org/10.1016/j.learninstruc.2007.09.011

Even, R. (1990). Subject-Matter Knowledge for Teaching and the case of functions. Educational Studies in Mathematics, 21, 521-544. https:// doi.org/10.1007/BF00315943

Even, R. (1993). Subject-matter knowledge and pedagogical content knowledge: prospective secondary teachers and the function concept. Journal for Research in Mathematics Education, 24(2), 94-116. https://doi.org/10.5951/ jresematheduc.24.2.0094

Font, V. (2011). Las funciones y la competencia disciplinar en la formación docente matemática. UNO. Revista de Didáctica de las Matemáticas, 56, 86-94.

Gagatsis, A., \& Shiakalli, M. (2004). Ability to translate from one representation of the concept of function to another and mathematical problem solving. Educational Psychology, 24(5), 645-657. https://doi. org/10.1080/0144341042000262953

Godino, J., \& Batanero, C. (1994). Significado institucional y personal de los objetos matemáticos. Recherches en Didactique des Mathématiques, 14(3), 325-355.

Godino, J. D., Batanero, C., \& Font, V. (2007). The onto-semiotic approach to research in mathematics education. ZDM. The International Journal on Mathematics Education, 39(1), 127-135. https://doi.org/10.1007/ s11858-006-0004-1

Godino, J., Giacomone, B., Font, V., \& Pino-Fan, L. (2018). Conocimientos profesionales en el diseño y gestión de una clase sobre semejanza de triángulos. Análisis con herramientas del modelo CCDM. Avances de Investigación en Educación Matemática, 13, 63-83. https:// doi.org/10.35763/aiem.v0i13
Heuvel-Panhuizen, M. (2020). Seen Through Other Eyes Opening Up New Vistas in Realistic Mathematics Education Through Visions and Experiences from Other Countries. In M. Heuvel-Panhuizen (Ed.), International Reflections on the Netherlands Didactics of Mathematics Visions on and Experiences with Realistic Mathematics Education, (pp. 1-20). Springer Open. https://doi. org/10.1007/978-3-030-20223-1_1

Hitt, F. (1998). Difficulties in the Articulation of Different Representations Linked to the Concept of Function. Journal of mathematical behavior, 17(1), 123-134. https://doi.org/10.1016/ S0732-3123(99)80064-9

Kaput, J. (1987). Representation systems and mathematics. In C. Janvier (Ed.), Problems of representation in the teaching and learning of mathematics (pp. 19-26). Hillsdale, NJ: Lawrence Erlbaum Associates.

Kaur, B., Wong, L. \& Govindani, S. (2020). Graphing Linear Equations-A Comparison of the Opportunity-to-Learn in Textbooks Using the Singapore and the Dutch Approaches to Teaching Equations. In M. HeuveL-Panhuizen (Ed.), International Reflections on the Netherlands Didactics of Mathematics Visions on and Experiences with Realistic Mathematics Education (pp. 97-111). Springer Open. https://doi.org/10.1007/978-3-030-20223-1_7

Kurnaz, M., \& Bayri, N. (2018). The Analysis of Secondary School Students' Transition Situations in Multiple Representations. Science Education International, 29(1), 3-10.

Maz, A. (2005). Los números negativos en España en los siglos XVIII y XIX [Tesis doctoral]. Universidad de Granada, Granada, España.

Ministerio de Educación. (2019). Texto definitivo bases curriculares $3^{\circ}$ y $4^{\circ}$ medio. Plan de Formación General Plan de Formación Diferenciada Humanístico-Científico. Ministerio de educación.

Mora, G., Riquelme, L., Troncoso, J., \& Escobar, B. (2018). Validación de una matriz para evaluar monografías en estudiantes universitarios. Formación Universitaria, 11(1), 63-76. http://dx.doi.org/10.4067/ S0718-50062018000100063.

Niss, M. \& Højgaard, T. (2019). Mathematical competencies revisited. Educational Studies in Mathematics, 102(1), 9-28. https://doi. org/10.1007/s10649-019-09903-9 
Nonaka, I., \& Toyama, R. (2015). The knowledge-creating theory revisited: knowledge creation as a synthesizing process. In J. S. Edwards (Eds.), The Essentials of Knowledge Management (pp. 2-10). OR Essentials Series. Palgrave Macmillan. https://doi. org/10.1057/9781137552105_4

Pino-Fan, L., \& Godino, J. (2015). Perspectiva ampliada del conocimiento didáctico-matemático del profesor. Paradigma, 36(1), 87-109.

Pino-Fan, L., Font, V., \& Breda, A. (2017). Mathematics teachers' knowledge and competences model based on the onto-semiotic approach. In B. Kaur, W. K. Ho, T. L. Toh \& B. H. Choy (Eds.), Proceedings of the 41st Conference of the International Group for the Psychology of Mathematics Education (Vol. 4, pp. 33-40). PME.

Pino-Fan, L., Parra-Urrea, Y., \& Castro, W. (2019). Significados de la función pretendidos por el currículo de matemáticas chileno. $\mathrm{Ma}$ gis, Revista Internacional de Investigación en Educación, 11(23), 201-220. https://doi. org/10.11144/Javeriana.m11-23.sfpc

Rau, M., Rummel, N., \& Aleven, V. (2017). Making connections among multiple graphical representations of fractions: sense-making competencies enhance perceptual fluency, but not vice versa. Instructional Science, 45(3), 331-357. https://doi.org/10.1007/ s11251-017-9403-7

Rey, G.; Boubée, C., Sastre, P., \& Cañibano, A. (2009). Ideas para enseñar. Aportes didácticos para abordar el concepto de función. Revista iberoamericana de educación matemática, 20, 153-162.

Rosa, M., \& Orey, D. (2011). Ethnomathematics: the cultural aspects of mathematics. Revista Latinoamericana de Etnomatemática, 4(2). 32-54.
Sastre, P.; Rey, G., \& Boubée, C. (2008). El concepto de función a través de la historia. Revista Iberoamericana de Educación Matemática, $16,141-155$.

Scherer, P. (2020). Low Achievers in Mathematics Ideas from the Netherlands for Developing a Competence-Oriented View. In M. Heuvel-Panhuizen (Ed.), International Reflections on the Netherlands Didactics of $\mathrm{Ma}$ thematics Visions on and Experiences with Realistic Mathematics Education (pp. 113132). Springer Open.

Schoenfeld, A. H. (1992). Learning to think mathematically: Problem solving, metacognition, and sense-making in mathematics. In D. Grouws (Ed.), Handbook for Research on Mathematics Teaching and Learning (pp. 334-370). MacMillan.

Schoenfeld, A., \& Kilpatrick, J. (2008). Towards a theory of proficiency in teaching mathematics. In D. TIROSH, T. WOOD (Ed.), Tools and Processes in Mathematics Teacher Education (321-354). Sense Publishers. https:// doi.org/10.1163/9789087905460_016

Siegler, R., Carpenter, T., Fennell, F., Geary, D., Lewis, J., Okamoto, Y., ... Wray, J. (2010). Developing effective fractions instruction: A practice guide. National Center for Education Evaluation and Regional Assistance, Institute of Education Sciences, U.S. Department of Education. https://doi. org/10.1007/978-3-030-20223-1_8

Wills, T., Shipley, T., Chang, B., Cromley, J., \& Booth, J. (2014). What Gaze Data Reveal About Coordinating Multiple Mathematical Representation. Journal Proceedings of the Annual Meeting of the Cognitive Science Society, 36(36), 312-3118.

\section{(c) $(1) \Theta$}

Competencias de profesores en formación en matemáticas al transformar las representaciones de una función (Tulio Amaya De Armas • Arjuna Gabriel Castellanos • Luis Roberto Pino-Fan) Uniciencia is protected by Attribution-NonCommercial-NoDerivs 3.0 Unported (CC BY-NC-ND 3.0) 